\title{
Wettbewerbsvorteile durch
}

\section{freiwillige Umweltberichterstattung}

\author{
Nicht nur Umweltverbände, sondern auch die Mitarbeiter, Kunden oder \\ Nachbarn verlangen zunehmend mehr Umweltinformationen von Unternehmen. \\ Doch wie wirkt sich freiwillige Umweltberichterstattung auf deren \\ Wettbewerbsfähigkeit aus?
}

$\mathrm{D}$

Von Klaus Fichter ie aktive Kommunikation über Umweltfragen hat in den vergangenen Jahren erheblich an Bedeutung gewonnen (vgl Abb. 1). Dies zeigen nicht zuletzt die rund 1.300 deutschen Unternehmen und Standorte, die bislang einen eigenständigen Umweltbericht veröffentlicht haben (1). Weltweit dürften bislang rund 2.500 Unternehmen oder Standorte die Öffentlichkeit mit einem eigenständigen Umweltbericht informieren.

Im Auftrag des Wirtschaftsministeriums des Landes Hessen hat das IöW Berlin zum ersten Mal den Nutzen einer freiwilligen Umweltberichterstattung von Unternehmen untersucht. Besonderes Augenmerk galt der bislang kaum thematisierten Frage, inwieweit Umweltberichterstattung aus Wettbewerbsgründen erfolgt und inwieweit sie sich auf die Wettbewerbsfähigkeit auswirkt (2). Die Untersuchung umfaßte eine schriftliche Befragung aller 500 deutschen Unternehmen und Standorte, die bis September 1996 einen Umweltbericht und/ oder eine Umwelterklärung nach der EG-Öko-Audit-Verordnung veröffentlicht hatten. Die Rücklaufquote betrug 46,8 Prozent. Aufbauend auf den Ergebnissen der schriftlichen Befragung wurden beim Pharma- und Chemiekonzern Hoechst (weltweit 150000 Mitarbeiter) und beim Babynahrungsmittelhersteller Hipp (750 Mitarbeiter) Fallanalysen durchgefiuhrt.

\section{Betrieblicher Nutzen}

Die Ergebnisse der Untersuchung stïtzen die Annahme, daß eine aktive Umweltberichterstattung die Wettbewerbsfähigkeit von Unternehmen in vielfältiger Hinsicht stärkt. Dabei hat die Berichterstattung nicht nur einen positiven Einfluß auf das Umweltimage der Unternehmen und die Beziehungen zu gesellschaftlichen Anspruchsgruppen, sondern trägt auch zur Pro- filierung am Markt bei. Außerdem fördert sie in deutlicher Weise die Qualität des Managements und des Umweltcontrolling und erhöht die Identifikation der Mitarbeiter mit dem Unternehmen.

Der hohe betriebliche Nutzen der bisherigen Umweltberichterstattung wird durch die Tatsache unterstrichen, daß 95 Prozent der befragten Unternehmen auch weiterhin Umweltberichte und Umwelterklärungen veröffentlichen wollen. Nur ein Prozent der befragten Unternehmen wollen dies nicht mehr tun. Dieses Ergebnis unterstützt die Prognose, daß sich die Umweltberichterstattung von Unternehmen in den kommenden Jahren weiter ausbreiten wird.

Aus Sicht der Unternehmen besteht eine der Hauptwirkungen der Umweltberichterstattung in der Förderung des Dialoges mit Meinungsführern und Multiplikatoren aus Umweltverbänden, Medien, Politik und Wissenschaft. 66 Prozent der Befragten sind der Meinung, daß dies für ihr Unternehmen zutrifft. Diese Zielgruppe hat ein besonderes Interesse an Überblicksinformationen zum Stand und an der Entwicklung des Umweltschutzes. Die Einschätzung des Nutzens einer freiwilligen Umweltberichterstattung differiert u.a. in Abhängigkeit von den spezifischen Informationsinteressen der jeweiligen Anspruchsgruppe. Die Fallanalysen zeigen, daß sich noch zwei weitere Hauptzielgruppen unterscheiden lassen:

- Die breite Öffentlichkeit und die Belegschaft, die vorrangig an kurzen, allgemeinverständlichen Informationen $\mathrm{zu}$ Themen oder Maßnahmen interessiert sind, die sie direkt betreffen.

- Spezialisten wie z.B. fachkundige Vertreter aus Bürgerinitiativen, Umweltgruppen, Behörden, Wissenschaft oder Umweltbeauftragte aus Unternehmen, die vorrangig Detailinformationen zu Einzelthemen oder Problemfragen wünschen.

Sowohl die Unternehmen als auch die verschiedenen Anspruchsgruppen betrachten die Umweltberichterstattung als nützlich, allerdings aus unterschiedlichen Motiven. Von der Nutzerseite her wird jedoch noch Standardisierungsbedarf gesehen und die Sicherstellung einer ausgewogenen Darstellung und die Vergleichbarkeit präsentierter Daten gefordert.

Nach Angaben der befragten Unternehmen besteht ein weiterer wesentlicher Nutzen der Umweltberichterstattung in der Verbesserung der verfügbaren Daten sowie der Planung und Erfolgskontrolle im Umweltschutz. Die Berichterstattung gibt wichtige Impulse zur Aufdeckung

\section{Abb. 1: Anzahl deutscher Unternehmen mit Umweltberichten bzw. Umwelterklärungen}

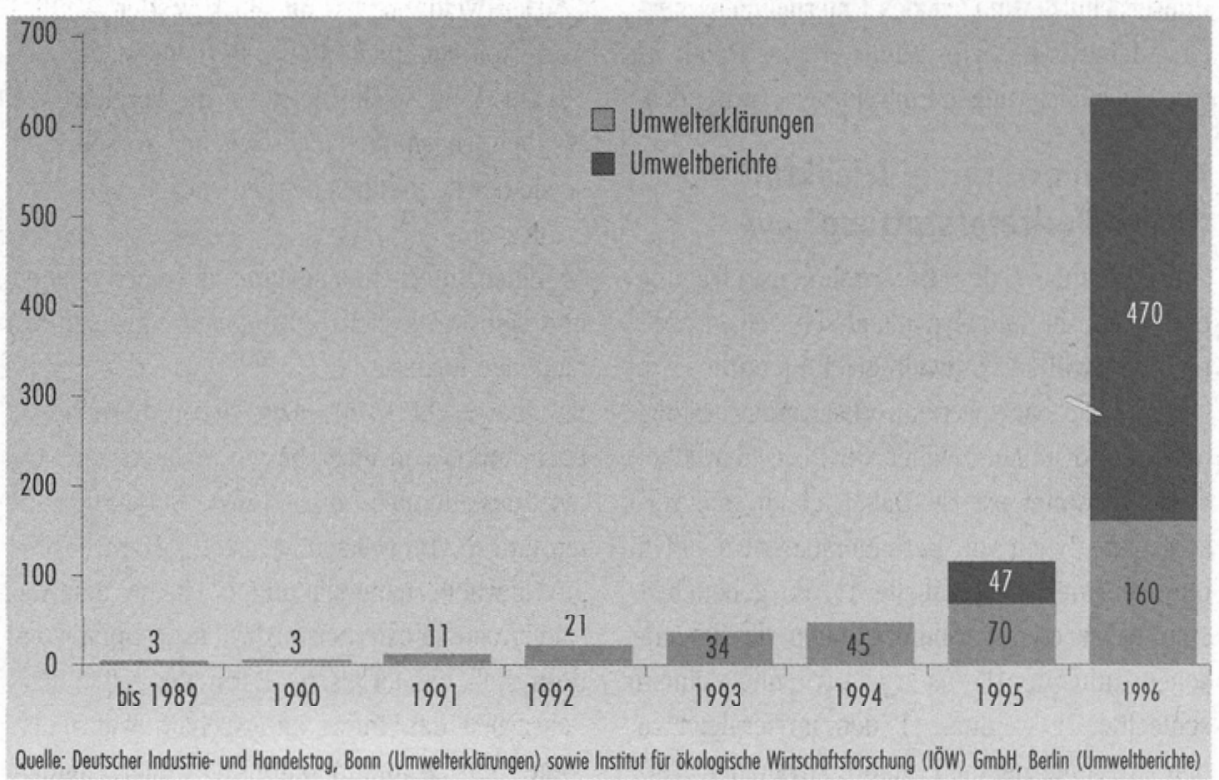


Tabelle 1:Verschiedene Berichterstattungstypen im Überblick

\begin{tabular}{|c|c|}
\hline & $\begin{array}{l}\text { Manas } \\
\text { orient }\end{array}$ \\
\hline Berichterstattungsfyp & $\begin{array}{l}\text { Umwelt- } \\
\text { controlling }\end{array}$ \\
\hline Houptaufgabe & $\begin{array}{l}\text { Unterstützung eines } \\
\text { systematischen Umwell- } \\
\text { controlling; Mitarbeiter- } \\
\text { motivation }\end{array}$ \\
\hline Wichtigste Zielgruppe & Mitorbeiter \\
\hline Art der Umweltberichterstattung & $\begin{array}{l}\text { mit Betriebsökobilanz, } \\
\text { problem- und daten- } \\
\text { orientiert; systematisch }\end{array}$ \\
\hline vorwiegend anzutreffen bei... & $\begin{array}{l}\text { mittelgrofe Unternehemen } \\
\text { (1000-2000 Mitarbeitern), } \\
\text { die in ihren Bronchen eine } \\
\text { Vorreiterrolle einnehmen }\end{array}$ \\
\hline Anteil & $10 \%$ \\
\hline
\end{tabular}

von Daten- und Bewertungslücken und trägt so zur kontinuierlichen Verbesserung der Umweltschutzleistung bei. Eine öffentliche Berichterstattung fördert also die Management- und Controllingqualität des Unternehmens.

60 Prozent der befragten Unternehmen stimmen der Aussage zu: „Durch den Umweltbericht und das damit verbundene öffentliche Bekenntnis zum Umweltschutz hat dieser einen höheren Stellenwert bei der Geschäftsführung bekommen.“ Die Umweltberichterstattung erhöht den Grad an Verbindlichkeit und die Selbstverpflichtung im Umweltschutz. Durch den öffentlichen Charakter der Berichterstattung ist diese zwangsläufig auch „Chefsache“. Die Notwendigkeit für die Geschäftsführung, sich mit den Umweltbelastungen und Umweltschutzleistungen des Unternehmens auseinanderzusetzen, wird damit erhöht. Damit ist eine Sensibilisierung in Umwelffragen verbunden.

\section{- Wettbewerb als Triebkraft der Berichterstattung}

Die Ergebnisse der Unternehmensbefragung und der Fall- und Typenanalysen zeigen, daß eine freiwillige Umweltberichterstattung in erster Linie aus Wettbewerbsgründen erfolgt und um so umfangreicher wird, desto wettbewerbsrelevanter sie ist. Dabei lassen sich verschiedene Typen von Berichterstattern deutlich unterscheiden (vgl. Tabelle 1). So geben beispielsweise die Großunternehmen der chemischen Industrie, die nach wie vor unter einem schlechten Umweltimage leiden, im Vergleich zu den Großunternehmen anderer Branchen trotz vergleichbarer Unternehmensgröße fast doppelt soviel Geld für ihre Berichte aus. Während die Unternehmen des Berichterstattungstyps „Imageprobleme“ im Durchschnitt 161.000 DM für einen Umweltbericht ausgeben, sind es beim Typ „Konzernkommunikation“ lediglich 88.000 DM. Die Befragung und die Fallanalysen stïtzen die Annahme, daß die Wettbewerbsrelevanz der Umweltberichterstattung deren Qualität bestimmt. Der Grad an Wahrheitstreue und Offenheit in der Berichterstattung hängt wesentlich von vier Faktoren und deren Zusammenwirken ab:

(1)Den Informationsansprüchen, die vom Markt, von der Öffentlichkeit oder von der Politik an das Unternehmen gestellt werden und damit dem ,Zwang zur Offenheit“.

(2)Der Wettbewerbsstrategie bzw. der Wettbewerbskompatibilität offener Berichterstattung.

(3)Der Umweltschutzleistung im Vergleich zu Wettbewerbern und zu gesetzlichen Vorschriften sowie der Konfliktbeladenheit betrieblicher Umweltfragen.

(4)Einer Unternehmenskultur, die einen offenen und selbstkritischen Umgang mit Umweltinformationen fördert.

Es überrascht nicht, daß sich Unternehmen nach außen von ihrer besten Seite zeigen wollen. Dies gilt auch für die Umweltschutzaktivitäten und die Umweltschutzleistung. Die freiwillige Umweltberichterstattung ist keine neutrale Unterrichtung externer Anspruchsgruppen, sondern stellt eine typische Form der Öffentlichkeitsarbeit dar. Informationsgehalt, Wahrheitstreue und Kommunikationsstil unterscheiden sich zwar deutlich von der Werbung, sind aber nichtsdestotrotz interessengeleitet. Während die Unternehmen eine leistungsorientierte Umweltberichterstattung betreiben, ist ein Großteil der externen Anspruchsgruppen an einer problemorientierten Darstellung interessiert.

50 Prozent der Unternehmen sind der Auffassung, daß sie durch die Umweltberichterstattung in puncto Umweltschutzleistung besser mit ihren Wettbewerbern vergleichbar geworden sind. Durch die Veröffentlichung von Umweltdaten werden die Grundlagen für mehr Transparenz und eine bessere Vergleichbarkeit geschaffen.

Die bisherigen Umweltberichte und Umwelterklärungen sind aber nur ein erster Schritt in diese Richtung, denn bislang fehlen ausreichend konkrete Standards, die eine Vergleichbarkeit gewährleisten würden. Dies wird nicht nur von Umweltverbänden, sondern auch vom Handel kritisiert. Große Handelsunternehmen wie Tengelmann erwägen, in der Zukunft Umweltberichte zu Vergleichen zwischen Lieferanten heranzuziehen. Auch die Vorgaben für die Umwelterklärung des EG-Öko-Audit-Systems sind zu allgemein gehalten, als daß eine tatsächliche Vergleichbarkeit gegeben wäre.

Die Wirkungen der Umweltberichterstattung von Unternehmen auf die Wettbewerbsfähigkeit sind vielfältig (vgl. Abb. 2). Die vorliegende Untersuchung zeigt, daß sie bislang keine erkennbaren wettbewerbsrelevanten Konflikte oder Nachteile für jene Unternehmen bewirkt hat, die eine aktive Umweltberichterstattung betreiben. Allerdings kann aus diesem Befund nicht gefolgert werden, 
daß eine aktive Umweltberichterstattung immer zur Stärkung der Wettbewerbsfähigkeit beiträgt. Bei der Bewertung der Praxiserfahrungen muß berücksichtigt werden, daß sich aufgrund fehlender bindender Standards Unternehmen bisher nach außen so darstellen können, daß es ihnen zum Vorteil gereicht. Außerdem kann ein Großteil der bislang berichterstattenden Unternehmen ein überdurchschnittliches Umweltschutzengagement vorweisen und mithilfe deren aktiver Kommunikation „Pioniergewinne“ erzielen. Auch wenn für den Zusammenhang zwischen Umweltberichterstattung und Wettbewerbsfähigkeit ein Konfliktmodell unangemessen ist, ist daher vor der Beschreibung als einfaches Harmoniemodell zu warnen.

\section{- Politische Schlußfolgerungen}

Mit der Umweltberichterstattung wird die Beurteilung der Umweltschutzleistung gesamter Unternehmen in marktliche Prozesse eingeführt. Die Berichterstattung generiert neue Informationstatbestände und neue Informationsflüsse. Auf diese Weise können Ineffizienzen in der Wertschöpfungskette aufgedeckt und innovative Lösungen gefördert werden. Umweltschutzkriterien und neue Informationen verändern den Selektionsmechanismus am Markt. Dies kommt bislang aber nur eingeschränkt zum Tragen, da fehlende Standards und informatorische Regelungen die Aussagekraft der Berichterstattung und die Vergleichbarkeit der Umweltschutzleistung noch erheblich einschränken. Außerdem mangelt es an professionellen Einrichtungen, die sich der gezielten Sammlung unternehmensbezogener Umweltinformationen widmen und diese für einzelne Nutzergruppen in anwendungsfähiger Form aufbereiten. Erste Ansätze dazu stellen z.B. Öko-Rating-Agenturen dar, die die Umweltschutzleistung gesamter Unternehmen bewerten und die Ergebnisse u.a. Banken, Fondsmanagern und institutionellen Anlegern zur

\section{Global Governance und Umweltpolitik}

ist das Thema des Informationsdienstes

\section{Ökologisches Wirtschaften 2/98}

Wenn Sie potentielle Beiträge haben, wenden Sie sich bitte an die Redaktion!
Abb. 2: Wirkung der Umweltberichterstattung auf die Wettbewerbsfähigkeit

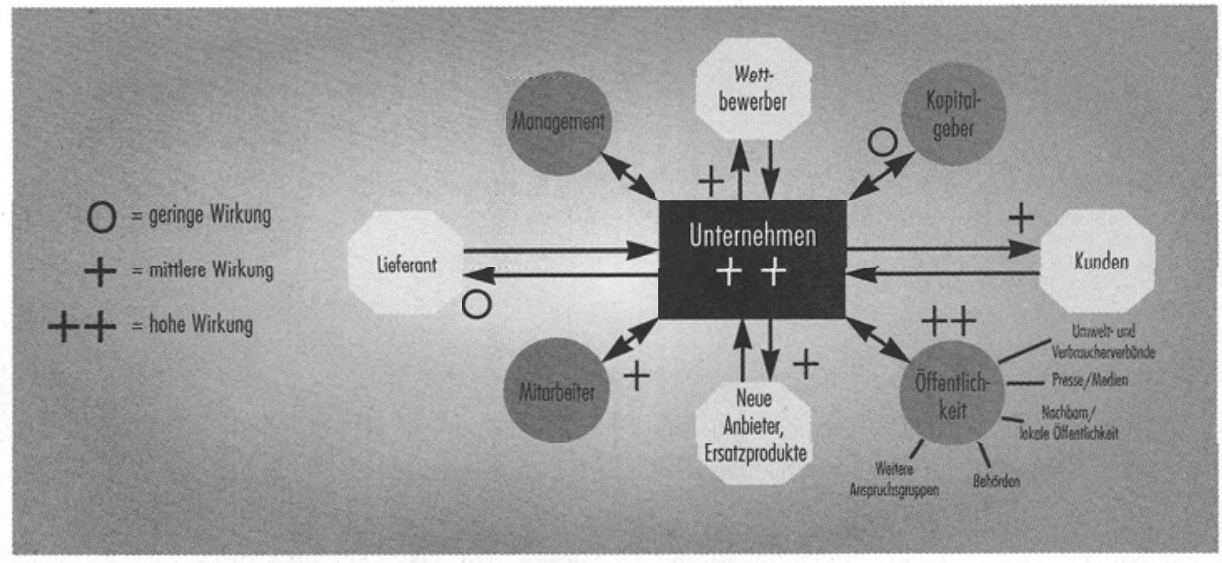

Verfügung stellen. Der Rechtsrahmen für einen funktionierenden Wettbewerb der Umweltschutzleistung von Unternehmen ist derzeit nicht gegeben.

Vor diesem Hintergrund ist eine gesetzliche Verankerung der Umweltberichterstattung von Unternehmen dringend geboten. Damit könnte eine größere Transparenz über die Umweltauswirkungen und Umweltschutzleistungen gesamter Unternehmen geschaffen werden. Außerdem würden damit Markttransparenz und -dynamik erhöht und bestehende Informationsasymmetrien zwischen Unternehmen und gesellschaftlichen Anspruchsgruppen abgebaut.

Eine verpflichtende Umweltberichterstattung sollte in Anlehnung an Publizitätspflichten in der Finanzberichterstattung an die Größe und Rechtsform von Unternehmen gekoppelt werden. Dies erscheint aus zweierlei Gründen sinnvoll: zum einen bestehen für umweltrelevante Anlagen und Standorte bereits zahlreiche Einzelberichtspflichten, eine gravierende Informationslïcke besteht derzeit vor allem bei anlagen- und standortübergreifenden Umweltinformationen. Zum anderen kann davon ausgegangen werden, daß Großunternehmen in besonderem Maße Umweltbelastungen verursachen und aufgrund ihres wirtschaftlichen Einflusses in besonderer Weise auf Stoff- und Energieströme, Umweltauswirkungen sowie Produktions- und Konsummuster Einfluß haben und daher umweltpolitisch besonders bedeutsam sind.

Eine Umweltberichterstattung, wie sie hier vorgeschlagen wird, setzt ein bestehendes Umweltcontrolling und Umweltmanagementsystem voraus. Unternehmen, die zu einer unternehmensbezogenen Umweltberichterstattung gesetzlich verpflichtet werden, müssen daher über ein Umweltmanagementsystem verfügen, das den Anforderungen der EG-Öko-Audit-Verordnung oder der DIN/ISO-
Norm 14001 „Umweltmanagementsysteme“ entspricht. Ein 1997 in den Niederlanden verabschiedetes Gesetz zur unternehmensbezogenen Umweltpflichtberichterstattung bindet diese ebenfalls an ein existierendes Umweltmanagementsystem. Vergleichbar mit der Jahresabschlußprüfung durch einen Wirtschaftsprüfer sollte ein Umweltbericht durch einen unabhängigen Sachverständigen auf Vollständigkeit, Wahrheit, Wesentlichkeit, Kontinuität und Vergleichbarkeit geprüft und validiert werden.

Eine unternehmensbezogene Umweltberichterstattung ist als Ergänzung zu bereits bestehenden Berichtspflichten zu konzipieren, da sie andere Zielgruppen anspricht. Sie wäre demnach als markt- und öffentlicheitsorientierte Unternehmenskommunikation $\mathrm{zu}$ verstehen. Wie die Untersuchung gezeigt hat, sind Umweltberichte nur sehr eingeschränkt für die breite Öffentlichkeit geeignet. Sie sollten daher nicht als Teil einer Breitenkommunikation, sondern als Spezialisten- und Multiplikatorenkommunikation konzipiert werden.

\section{Anmerkungen}

(1) Bei rund 1000 dieser Berichte handelt es sich um Umwelterklärungen von Standorten, die am EG-0̈ko-AuditSystem teilnehmen.

(2) Die ausführlichen Ergebnisse enthölt der wissenschaftliche Endbericht des Projektes: Klaus Fichter, Thomas Loew: Wettbewerbsvorteile durch Umweltberichterstattung, IÖWSchriftenreihe 119/97, Berlin, 1997.

Der Autor
Klaus Fichter ist wissenschaftlicher Mitarbeiter am
Institut für ökologische Wirtschaftsforschung, Berlin.
Kontakt: I0̈W, Giesebrechtstr. 13, 10629 Berlin,
Tel.030/8845940, Fax 030/8825439.
e-mail: mailbox@ioew.b.eunet.de


(c) 20I0 Authors; licensee IÖW and oekom verlag. This is an article distributed under the terms of the Creative Commons Attribution Non-Commercial No Derivates License (http://creativecommons.org/licenses/by-nc-nd/3.o/), which permits unrestricted use, distribution, and reproduction in any medium, provided the original work is properly cited. 